\title{
A single double-strand break system reveals repair dynamics and mechanisms in heterochromatin and euchromatin
}

\author{
Aniek Janssen, ${ }^{1}$ Gregory A. Breuer, ${ }^{2,3}$ Eva K. Brinkman, ${ }^{4}$ Annelot I. van der Meulen, ${ }^{1}$ Sean V. Borden, ${ }^{1}$ \\ Bas van Steensel, ${ }^{4}$ Ranjit S. Bindra, ${ }^{2,3}$ Jeannine R. LaRocque, ${ }^{5}$ and Gary H. Karpen ${ }^{1,6}$ \\ ${ }^{1}$ Biological Systems and Engineering Division, Lawrence Berkeley National Laboratory, Berkeley, California 94720, USA; \\ ${ }^{2}$ Department of Therapeutic Radiology, ${ }^{3}$ Department of Experimental Pathology, Yale School of Medicine, New Haven, \\ Connecticut 06510, USA; ${ }^{4}$ Division of Gene Regulation, Netherlands Cancer Institute, Amsterdam 1066 CX, the Netherlands; \\ ${ }^{5}$ Department of Human Science, School of Nursing and Health Studies, Georgetown University Medical Center, Washington, DC \\ 20057, USA; ${ }^{6}$ Department of Molecular and Cell Biology, University of California at Berkeley, Berkeley, California 94720, USA
}

Repair of DNA double-strand breaks (DSBs) must be properly orchestrated in diverse chromatin regions to maintain genome stability. The choice between two main DSB repair pathways, nonhomologous end-joining (NHEJ) and homologous recombination (HR), is regulated by the cell cycle as well as chromatin context. Pericentromeric heterochromatin forms a distinct nuclear domain that is enriched for repetitive DNA sequences that pose significant challenges for genome stability. Heterochromatic DSBs display specialized temporal and spatial dynamics that differ from euchromatic DSBs. Although HR is thought to be the main pathway used to repair heterochromatic DSBs, direct tests of this hypothesis are lacking. Here, we developed an in vivo single DSB system for both heterochromatic and euchromatic loci in Drosophila melanogaster. Live imaging of single DSBs in larval imaginal discs recapitulates the spatio-temporal dynamics observed for irradiation (IR)-induced breaks in cell culture. Importantly, live imaging and sequence analysis of repair products reveal that DSBs in euchromatin and heterochromatin are repaired with similar kinetics, employ both NHEJ and HR, and can use homologous chromosomes as an HR template. This direct analysis reveals important insights into heterochromatin DSB repair in animal tissues and provides a foundation for further explorations of repair mechanisms in different chromatin domains.

[Keywords: heterochromatin; recombination repair; cell cycle; homolog; NHEJ; Drosophila]

Supplemental material is available for this article.

Received April 21, 2016; revised version accepted July 5, 2016.

The eukaryotic nucleus contains distinct chromatin domains called heterochromatin and euchromatin (Heitz 1928). Constitutive heterochromatin is enriched for repetitive DNAs and contains few protein-coding genes. In contrast, euchromatin is generally associated with more open chromatin regions and contains many actively transcribed genes. Heterochromatin is predominantly concentrated at pericentromeric and telomeric regions, and disruption of heterochromatin impairs chromosome segregation, replication timing, transposon silencing, and gene expression (Weiler and Wakimoto 1995; Peters et al. 2001; Peng and Karpen 2009; Rangan et al. 2011). Epigenetically, heterochromatin is enriched for dimethylation and trimethylation of histone $\mathrm{H} 3$ Lys9 (H3K9me2/3) and its binding partner, heterochromatin protein 1 (HP1) (Eissenberg and Elgin 2000).

Corresponding authors: ghkarpen@lbl.gov, jan.larocque@georgetown.edu Article is online at http://www.genesdev.org/cgi/doi/10.1101/gad.283028. 116.
Changes in H3K9 methylation levels and distributions are seen in aging (Chen et al. 2014) and cancer (Sulli et al. 2012; Ellinger et al. 2014). Moreover, H3K9me2rich genomic regions are highly correlated with increased mutation load in a variety of cancer types (Schuster-Bockler and Lehner 2012), suggesting that heterochromatin regions are more susceptible to DNA damage and improper repair. Thus, determining how DNA repair in heterochromatic DNA is regulated will elucidate how chromatin states impact genome stability and how misregulation contributes to disease progression.

One of the most deleterious DNA lesions is a doublestrand break (DSB), since improper DSB repair can lead to formation of aberrant chromosomes (e.g., translocations and insertions) that contribute to cancer and

(C) 2016 Janssen et al. This article is distributed exclusively by Cold Spring Harbor Laboratory Press for the first six months after the full-issue publication date (see http://genesdev.cshlp.org/site/misc/terms.xhtml). After six months, it is available under a Creative Commons License (Attribution-NonCommercial 4.0 International), as described at http://creativecommons.org/licenses/by-nc/4.0/. 
developmental diseases (Janssen and Medema 2013). DSBs can occur during endogenous processes, such as replication fork collapse upon encountering an unrepaired DNA lesion, or by exogenous mutagens such as irradiation (IR) (Ciccia and Elledge 2010). The two main DSB repair pathways are homologous recombination (HR), in which a homologous template is used to accurately repair the DSB, and the more error-prone nonhomologous endjoining (NHEJ) pathway, in which two broken DNA ends are ligated together, often resulting in modifications of bases at the break site. HR is mainly limited to the $S$ and G2 cell cycle phases, when a sister chromatid is present and can be used as a recombination template. In contrast, NHEJ can be used at any stage of the cell cycle /Ciccia and Elledge 2010).

The importance of specific chromatin modifications and remodelers in DSB repair and pathway choice has become increasingly clear over the past decade (Price and D'Andrea 2013). For example, H3K36me3 at actively transcribed regions is associated with a preference for $\mathrm{HR}$ repair (Aymard et al. 2014), and specific chromatin environments can predispose a genome to translocations (Burman et al. 2015) or repair by the error-prone alternative NHEJ (Lemaitre et al. 2014).

DSBs in constitutive heterochromatin are considered especially dangerous due to the presence of many homologous repetitive sequences on different chromosomes. HR repair of damaged repeats can result in aberrant recombination products that are harmful for cells and organisms (Chiolo et al. 2011; Ryu et al. 2015). However, studies in mammalian and Drosophila melanogaster cultured cells do suggest that HR is the main repair pathway used by heterochromatic DSBs (Goodarzi et al. 2008; Chiolo et al. 2011; Goodarzi and Jeggo 2012). Heterochromatic DSBs display specific temporal and spatial responses that differ significantly from euchromatic DSBs. In Drosophila and mammalian cell culture, heterochromatic DSBs induced by IR relocalize to outside the heterochromatin domain (Chiolo et al. 2011; Jakob et al. 2011). 5' -to-3' end resection, phosphorylation of H2Av by ATM/ATR kinases $(\gamma \mathrm{H} 2 \mathrm{Av}$, analogous to $\gamma \mathrm{H} 2 \mathrm{AX}$ in mammals) (Rogakou et al. 1999), and recruitment of proteins that regulate early events in HR repair (e.g., ATRIP and TopBP1) occur at heterochromatic DSBs inside the domain within minutes after IR. However, proteins involved in late HR events (e.g., BRCA2, Rad51) are recruited to DSBs only after they relocalize (Chiolo et al. 2011). We hypothesized that these spatial and temporal dynamics help prevent aberrant recombination events between repetitive regions on nonhomologous chromosomes and promote "safe" recombination between sister chromatids or homologs. However, it is currently unclear whether heterochromatin-specific DSB relocalization to the nuclear periphery depends on induction of many breaks at the same time (using IR) or whether a single break induces the same dynamic behaviors.

A key role for HR was also demonstrated by genetic analyses. Depletion of HR proteins in Drosophila cells, but not NHEJ proteins, resulted in retention of IR-induced repair foci inside the heterochromatin domain /Chiolo et al. 2011). However, direct determination of all DSB repair pathways used as well as information about the templates used for HR require sequence analysis of repair products (Nagel et al. 2014; Soong et al. 2015), which is difficult for repetitive DNA. Thus, other pathways, such as NHEJ or single-strand annealing (SSA), could also play an important role in repairing heterochromatic DSBs. In fact, utilization of NHEJ would eliminate some aberrant repair events that result from HR between repetitive regions. In SSA, extensive end resection results in annealing of complementary repetitive sequences, which could be of particular importance in repeat-rich heterochromatin. Determining whether the NHEJ and SSA pathways impact heterochromatin DSB repair is important to understand the mechanisms that maintain the stability of repeated DNAs.

To address these questions, we developed a $D$. melanogaster single DSB system to analyze repair in both constitutive heterochromatic and euchromatic sites. Drosophila is an attractive system to study DSB repair in the context of a living organism with well-characterized chromatin environments (Kharchenko et al. 2011), effective tools for analyzing repair processes (Rong and Golic 2003; Preston et al. 2006; Do et al. 2014) and conservation of heterochromatin regulation and protein complexes with mammals (Fodor et al. 2010; Hoskins et al. 2015).

Here we show that single heterochromatic DSBs relocalize from the heterochromatin domain in living tissues and show repair kinetics similar to those of euchromatic DSBs. Most importantly, in contrast to earlier findings, genetic as well as sequence analyses revealed that NHEJ, SSA, and HR pathways are used to repair heterochromatic DSBs at frequencies similar to euchromatic DSBs. Finally, we developed an in vivo homolog-tracking system that demonstrates that both euchromatic and heterochromatic DSBs can use homologs as a template for HR repair. These findings advance our knowledge of the components and mechanisms that repair heterochromatic DSBs and ensure genome stability.

\section{Results}

Development of a single DSB system for heterochromatic and euchromatic loci

We developed a single DSB system to cytologically compare DNA damage repair in euchromatic and constitutive heterochromatic loci in animal tissues and determine pathway utilization by DNA sequence analysis of DSB repair products. Specifically, we used the Minos-mediated integration cassette (MiMIC) system (Venken et al. 2011) to integrate the DR-white (direct repeat white) DSB reporter (Do et al. 2014) into six pericentromeric heterochromatic and three euchromatic loci (Fig. 1A,B).

The DR-white reporter has one upstream nonfunctional white gene (white expression results in red eye color) containing an I-SceI recognition sequence, a red fluorescent marker (3xp3-dsRed), and a downstream truncated nonfunctional white gene (iwhite) (Fig. 1A). dsRed and white are driven by the eye tissue-specific promoter $3 x P 3$ and 
A
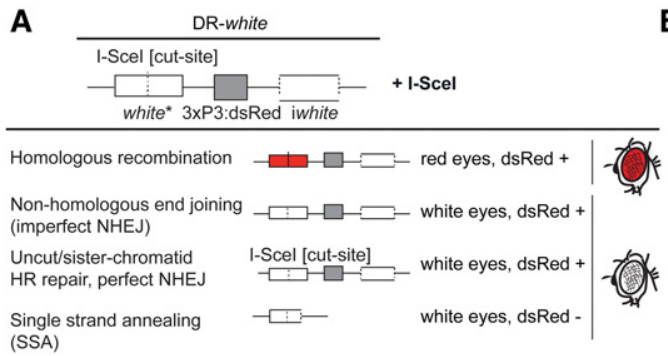

Single sta
(SSA)

D

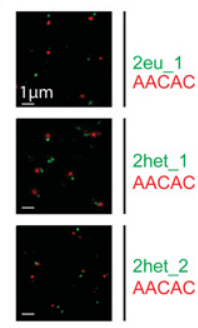

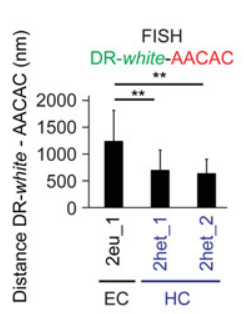

E

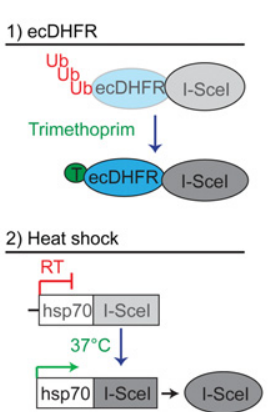

B

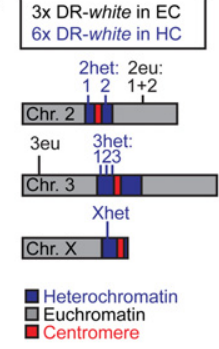

C
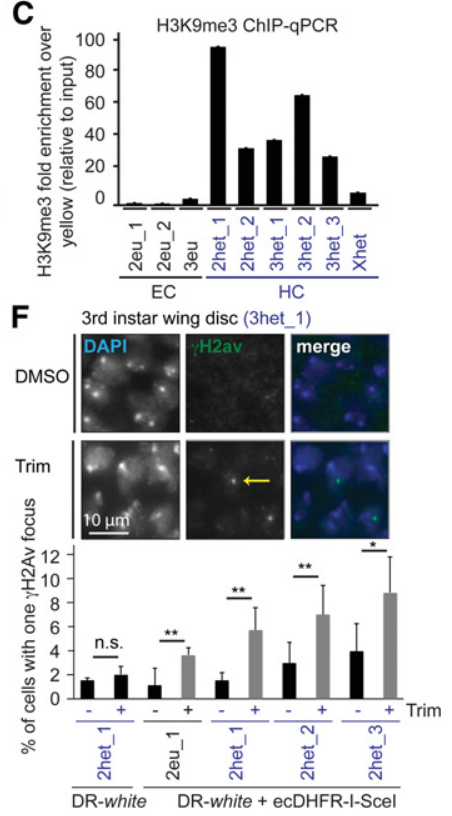

Figure 1. Inducible single-break system for specific heterochromatic and euchromatic sites. $(A)$ Schematic of the DR-white system. I-SceI expression produces a single DSB in the upstream white gene. HR with the downstream truncated iwhite sequence converts the 18-basepair (bp) I-SceI cut site to a wild-type white sequence (red eyes). White-eyed flies result from the absence of an I-SceI-induced DSB, NHEJ (perfect NHEJ or NHEJ with processing), HR repair using the sister chromatid, or SSA (with loss of the intervening 3xP3.dsRed marker), which can be analyzed in more detail by PCR and/or sequencing of repair products. $(B)$ Schematic of DR-white integrations in euchromatin (EC, eu) and heterochromatin (HC, het) on chromosomes 2, 3, and X. (C) ChIP-qPCR /chromatin immunoprecipitation [ChIP] followed by quantitative PCR [qPCR]) analysis of $\mathrm{H} 3 \mathrm{~K} 9 \mathrm{me} 3$ levels at the nine DR-white insertions. The graph shows average $\mathrm{H} 3 \mathrm{~K} 9 \mathrm{me} 3 \mathrm{enrichment}$ over input by qPCR (+SD) of three experiments using a primer set specific for the I-SceI cut site. H3K9me3 levels were standardized using a yellow qPCR primer set as an internal control (set at 1$)$. (D, left) Representative images of FISH staining for the AACAC heterochromatic repeat (red) and the DR-white locus (green) in third instar larval discs with the indicated genotypes. (Right) Quantification of FISH images. Each bar indicates average distance (in nanometers) between the AACAC and DR-white centroids + SD. $n \geq 200$ cells. $\left.{ }^{* *}\right) P$-value $\leq 1 \times 10^{-22}$, two-tailed unpaired Student's $t$-test. $(E)$ Schematic of two inducible I-SceI expression systems. (1) ecDHFR-I-SceI degradation through proteolysis is blocked upon addition of the stabilizing ligand trimethoprim. (2) The hsp70 promoter upstream of the I-SceI gene is activated by shifting for $1 \mathrm{~h}$ to $37^{\circ} \mathrm{C}$. (RT) Room temperature. (F, top) Representative images of immunofluorescence staining for $\gamma \mathrm{H} 2 \mathrm{Av}$ (green) in third instar larval wing disc cells. (Blue) DAPI(DNA). (Bottom) Quantification of the percentages (+SD) of nuclei that contain one $\gamma \mathrm{H} 2 \mathrm{Av}$ focus is plotted for samples treated for $3 \mathrm{~h}$ with the DMSO control (black bars) or trimethoprim (gray bars). $n \geq 4$ independent experiments, $\geq 500$ wing or leg disc cells each. $\left({ }^{*}\right) P$-value $\leq 0.05$; $\left({ }^{* *}\right) P$-value $\leq 0.002$; (n.s.) not significant $(P=0.2)$, two-tailed unpaired Student's $t$-test.

glass multiple reporter $(G M R)$, respectively. The upstream white gene contains a premature stop codon present in the I-SceI recognition sequence (Do et al. 2014). Expression of the rare cutting endonuclease I-SceI, used extensively in studies of DSB repair (Jasin 1996), induces a single DSB at the I-SceI cut site. Repair of this single DSB by HR, NHEJ, and SSA occurs in the germline as well as somatic cells. Relative contributions of each of these pathways can be quantitated by determining the phenotypes of the progeny (premeiotic male germline repair events) or by PCR and sequence analysis (somatic and germline repair events) (Fig. 1A).

We confirmed integration of all DR-white constructs in the targeted genomic loci (Fig. 1B) using PCR. In addition, we performed ChIP-qPCR (chromatin immunoprecipitation [ChIP] followed by quantitative PCR [qPCR]) using an antibody that specifically recognizes $\mathrm{H} 3 \mathrm{~K} 9 \mathrm{me}$, the key heterochromatin-enriched histone modification (Fig. 1C). As expected, the three euchromatic DR-white inser- tions were not enriched for $\mathrm{H} 3 \mathrm{~K} 9 \mathrm{me} 3$, whereas the six heterochromatic DR-white insertions showed an eightfold to 90 -fold enrichment of H3K9me3 at the I-SceI cut site compared with input (Fig. 1C). H3K36me3 has been linked to DSB repair pathway choice (Aymard et al. 2014), and introduction of DR-white insertions could potentially affect this transcription-associated mark. However, the expression of dsRed is under the control of an eye tissue-specific driver, and we observed strong silencing of $d s R e d$ in all heterochromatic DR-white insertions (data not shown). In addition, ChIP-qPCR analysis showed no change in H3K36me3 enrichment at DR-white insertions in comparison with modENCODE ChIP-seq data from wild-type larval tissue (Supplemental Fig. S1A). Finally, fluorescence in situ hybridization (FISH) on fixed larval imaginal discs with probes recognizing DR-white and the AACAC repeat, present in chromosome $2 \mathrm{R}$ heterochromatin, cytologically validated the DR-white integrations in heterochromatin. Consistent with the 
observed H3K9me3 patterns, heterochromatic DR-white integrations were spatially close $(500 \mathrm{~nm})$ to the AACAC repeat, whereas the euchromatic DR-white insertion on chromosome 2 was, on average, $1200 \mathrm{~nm}$ separated from the AACAC repeat (Fig. 1D).

In order to temporally control DSB formation, we used two different inducible I-SceI expression systems (Fig. 1E). Fusing I-SceI to an ecDHFR degradation domain (ecDHFR-I-SceI) allows I-SceI protein stabilization after addition of the ligand trimethoprim (Cho et al. 2013). Hsp70.I-SceI is a heat-shock-inducible system with hsp70 promoter-dependent I-SceI expression (Fig. 1E; Rong and Golic 2003). Three hours after incubating larval imaginal discs containing both ecDHFR-I-SceI and DRwhite in medium containing trimethoprim, $4 \%-8 \%$ of cells showed one $\gamma \mathrm{H} 2 \mathrm{Av}$ focus compared with $1 \%-3 \%$ in controls (DMSO only) (Fig. 1F), indicating that single DSBs can be temporally induced using the ligand-dependent system. Expression of ecDHFR-I-SceI after feeding larvae trimethoprim did not produce visible cell cycle defects or reduce organismal or cell viability, ruling out specific cell cycle or lethality-associated effects of the ecDHR-I-SceI system (Supplemental Fig. S1B-D). The hsp 70.I-SceI transgene was more efficient at inducing single DSBs, since $13 \%-20 \%$ of imaginal disc cells contained a single $\gamma \mathrm{H} 2 \mathrm{Av}$ focus $6 \mathrm{~h}$ after heat shock compared with $4 \%$ in control tissue (no heat shock) (Supplemental Fig. S1E). We conclude that both systems temporally induce single DSBs at DR-white loci. In addition, after induction, there were no consistent differences in $\gamma \mathrm{H} 2 \mathrm{Av}$ foci numbers between euchromatic and heterochromatic loci (Fig. 1F; Supplemental Fig. S1E), ruling out the possibility that heterochromatin regions are less accessible to cleavage by I-SceI.

\section{Live imaging reveals dynamic movement of single DNA damage foci in heterochromatin}

We previously discovered that IR-induced DNA damage foci in heterochromatin move to the periphery of the domain in Drosophila cultured cells (Chiolo et al. 2011). To determine whether similar movements occur after single DSB induction in vivo, we generated DR-white fly lines that express ecDHFR-I-SceI and red fluorescent protein (RFP)-tagged HP1a (marks the heterochromatin domain). To visualize DSBs, these flies also express mu2 tagged with enhanced yellow fluorescent protein (eYFP); mu2, the Drosophila ortholog of mammalian $\mathrm{MDC} 1$, binds to $\gamma \mathrm{H} 2 \mathrm{Av}$ and is recruited to DSB sites early in repair (Fig. 2A; Stucki et al. 2005; Dronamraju and Mason 2009).

We tracked the nuclear localization of eYFP-mu2 focus appearance and disappearance with respect to the RFPHP1a heterochromatin domain (Fig. 2A). As expected, the majority $(\sim 80 \%)$ of mu2 foci in cells containing euchromatic DR-white insertions first appear outside of the HP1a domain. In contrast, $\sim 70 \%$ of foci in cells containing heterochromatic insertions first appear inside or at the periphery of the HP1a domain (Fig. 2A). Ten percent to $15 \%$ of mu2 foci associated with DSBs at heterochro- matic DR-white insertions arose inside the HPla domain and moved to the domain periphery within one time frame (10 $\mathrm{min})$, where they were subsequently resolved; i.e., disappeared (Fig. 2A, dark-blue bars). Thirty percent to $50 \%$ of mu2 foci in cells containing heterochromatic DR-white insertions appeared at the periphery and stayed there until the mu2 focus resolved (Fig. 2A, red bars). This behavior likely reflects our inability to capture initial mu2 focus localization inside the HP1a domain due to low time resolution (one image every $10 \mathrm{~min}$ ). Alternatively, some of the uncut DR-white insertions could reside at the HP1a domain periphery, making it difficult to see the spatial dynamics observed for foci originating within the HP1a domain. Regardless, we conclude that the temporal and spatial relocation dynamics for I-SceI-induced single breaks in live tissues recapitulated our previous observations for IR-induced foci in cultured Drosophila cells (Chiolo et al. 2011). Interestingly, we also observed a small subset of foci $(5 \%-10 \%)$ that first appeared in the HP1a domain and were resolved without peripheral movement. This suggests that a small subset of heterochromatic DSBs does not move to the periphery and could complete repair within the heterochromatin domain.

In addition to data on the spatial movement of mu2 foci, live imaging also allowed direct assessment of the kinetics of mu2 focus appearance (onset of DSB repair) and disappearance (resolution of repair foci; this is not an absolute measure of the repair timing due to the possible persistence of $\gamma \mathrm{H} 2 \mathrm{Av}$ and mu2 after repair is finished) (Mah et al. 2010). Studies using IR in mammalian cells suggested that heterochromatic DSBs display slower repair rates compared with euchromatic DSBs /Goodarzi et al. 2008; Beucher et al. 2009|. However, we found that the average time required to resolve mu2 foci was not significantly different between three heterochromatic and three euchromatic insertions (Fig. 2B). DSBs in both chromatin regions showed similar kinetics; $50 \%$ of mu2 foci disappeared within $60 \mathrm{~min}$ after appearance, and the time from appearance to disappearance displayed a wide range in the remaining $50 \%$ of foci, from $\sim 60$ to $>350$ min (Fig. 2B). We conclude that although there are sitespecific differences in mu2 foci kinetics (Fig. 2, cf. D controls and E controls [black lines]), there are no significant differences in the average rate of $\mathrm{mu} 2$ focus disappearance at euchromatic versus heterochromatic loci, in contrast to previous findings (Goodarzi et al. 2008).

\section{Live imaging reveals that disruption of HR or NHEJ pathways delays repair kinetics at both heterochromatic and euchromatic DSBs}

HR has been reported to be the major pathway responsible for repair of DSBs in heterochromatin (Beucher et al. 2009; Chiolo et al. 2011). We previously found that depletion of DmRad51 (HR protein, Rad51, encoded by spn-A) in Drosophila cultured cells, but not depletion of DmKu70 (encoded by Irbp) or DmKu80 (encoded by Ku80) (NHEJ proteins), resulted in defective relocalization and aberrant accumulation of DNA damage foci within heterochromatin following IR (Chiolo et al. 2011). 
A
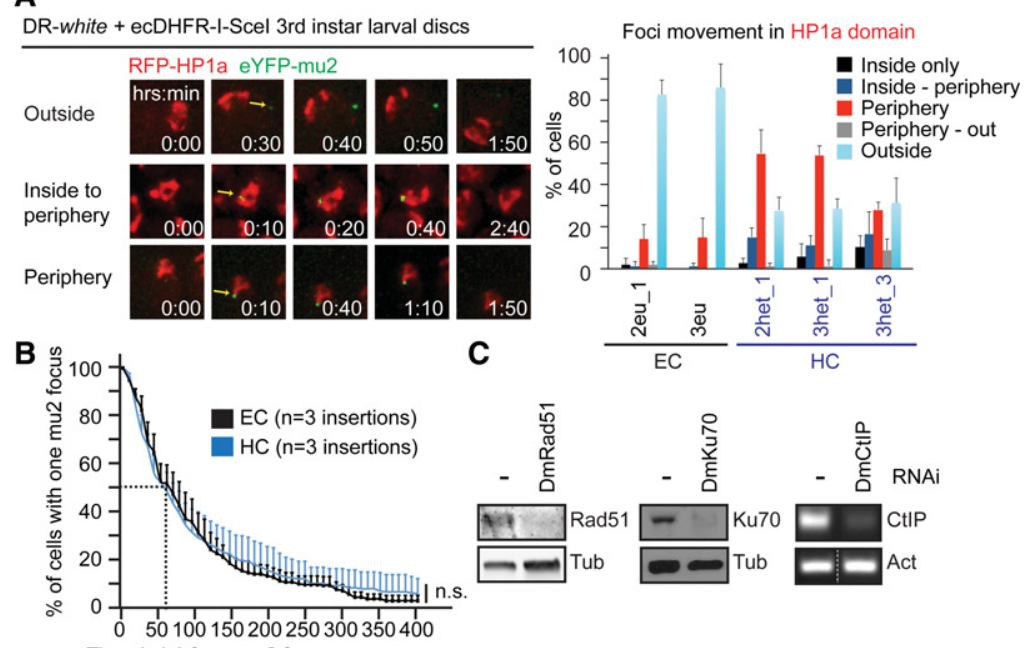

D Time ( $\mathrm{min})$ from mu2 focus appearance
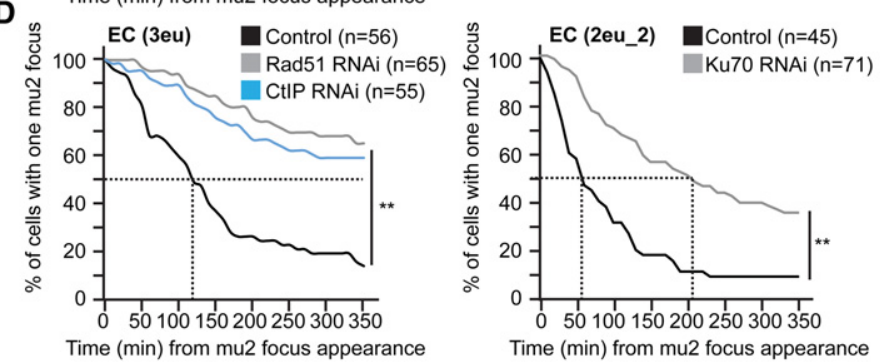

E
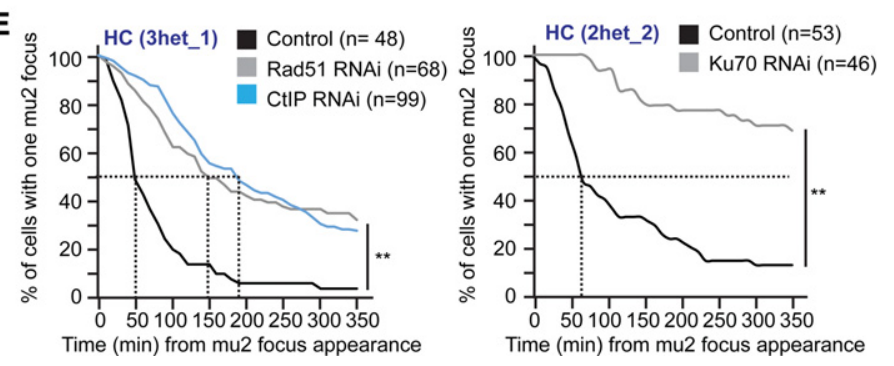

Figure 2. Live imaging of single-break dynamics in euchromatin and heterochromatin. $(A$, left $)$ Representative images of mu2-YFP focus dynamics (green) with respect to the HP1a domain (red) in larval wing disc cells. The three most common categories of mu2 focus dynamics are shown: (1) arise and resolve outside of the HP1a domain (top), (2) arise inside the HP1a domain and move to the periphery (middle), and (3) arise and resolve at the HP1a periphery (bottom). (Right) Quantification and categorization of single mu2 focus dynamics. Bars indicate the average percentage $(+\mathrm{SD})$ of three independent experiments per DR-white insertion. $n=30$ cells of leg and wing discs imaged per experiment. (B) Time-lapse analysis of single mu2-YFP focus disappearance (minutes from appearance) in third instar larval leg and wing discs treated as in $A$. The time point of mu2 focus appearance was set at $t=0$ for each individual focus. $n=$ number of DR-white insertions imaged for either heterochromatin (HC) or euchromatin (EC); at least 90 cells (=single mu2 focus) were analyzed per insertion. Error bars indicate +SEM. (n.s.) $P$-value $=$ 0.2295, log-rank (Mantel-Cox) test. Dashed line indicates the time it took for $50 \%$ of mu 2 foci to disappear. (C) Lysates from Actin-GAL4-expressing $(-)$ or Actin-GAL4 and UAS.DmRad51-expressing (left) or UAS.DmKu70 RNAi-expressing (middle) third instar larvae were immunoblotted for tubulin and DmRad51 (left) or DmKu70 (middle). (Right) RT-PCR with DmCtIP and actinin-specific primers on RNA from third instar larvae expressing ActinGAL4 (-) or Actin-GAL4 and UAS.DmCtIP RNAi. $(D, E)$ Wing and leg disc cells with the indicated RNAi depletions were imaged in the presence (black lines; GAL4 only) or absence of either DmRad51 (gray line; left graphs), DmCtIP (blue line; left graphs), or DmKu70 (gray line; right graphs) as in $B$. (n) Number of cells imaged per

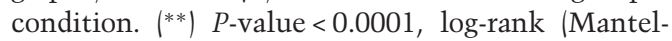
Cox) test. Different euchromatin and heterochromatin DR-white integrations were imaged with DmRad51/DmCtIP or DmKu70 RNAi due to genetic limitations. Dashed lines indicate the time it took for $50 \%$ of mu2 foci to disappear.

To more directly analyze the impact of HR and NHEJ pathways on repair of I-SceI-induced single breaks in vivo, we depleted HR proteins DmRad51 or DmCtIP/ CG5872 (required for initiating $5^{\prime}$-to-3' end resection) (You and Bailis 2010) or the NHEJ protein DmKu70 by RNAi (Fig. 2C-E). Live analysis revealed that knockdown of DmRad51, DmCtIP (Fig. 2D,E left), or DmKu70 (Fig. $2 \mathrm{D}$, E right) resulted in significant delays in mu2 focus resolution for both euchromatic (Fig. 2D) and heterochromatic DSBs (Fig. 2E). For example, we observed that after DSB induction at the heterochromatic site 3het_1, 50\% of mu2 foci in controls resolved within $50 \mathrm{~min}$, which increased to 150 and 200 min upon DmRad51 or DmCtIP knockdown, respectively (Fig. 2E left). We also investigated whether the absence of HR or NHEJ repair caused defects in the relocalization of single heterochromatic DSBs. Depletion of DmRad51, DmCtIP, or DmKu70 did not re- sult in detectable DSB relocalization defects in live analysis (Supplemental Fig. S2A), although it is possible that more subtle effects were missed due to the limited temporal resolution of this analysis. DmKu70 or DmRad51 knockdown did not alter cell cycle progression in the absence of single breaks, indicating that the effects on foci kinetics are not due to DmKu70 or DmRad51 RNAiinduced cell cycle delays before break induction (Supplemental Fig. S2B). In addition, RNAi-mediated depletion of vermillion, a protein involved in Drosophila eye pigmentation, did not result in a significant delay in mu2 focus disappearance, ruling out the possibility that RNAi pathway activation is responsible for the observed changes in mu2 focus kinetics (Supplemental Fig. S2C). We conclude that, in contrast to previous studies, both HR and NHEJ proteins are required for DSB repair in both chromatin environments. 
Repair product analysis reveals the use of $H R$, SSA, and NHEJ repair pathways in heterochromatin

In order to more directly determine which pathways play a role in heterochromatin DSB repair, we leveraged the DRwhite system to quantitate the frequencies of different DSB repair products in the male germline (Figs. 1A, 3A; Do et al. 2014). Single DSBs were induced in both premeiotic germ cells and somatic cells by exposing DR-white/ hsp70.I-SceI embryos and larvae to heat shock (Do et al. 2014). To assess the frequencies of repair pathway utilization in the germline, adult males containing DR-white and hsp70.I-SceI were crossed with control females: Red-eyed DR-white progeny indicate an HR repair event in the paternal male germline (Figs. 1A, 3A). As expected, we observed HR repair events for all of the DR-white insertions, both heterochromatic and euchromatic (Fig. 3A). Despite suppression of white gene expression in the heterochromatic DR-white insertions (Fig. 3A, cf. right and left eyes of F0 generation), red-eyed progeny could still be quantified for both euchromatin and heterochromatin. The percentages of red-eyed progeny varied from $10 \%$ to $30 \%$ among the different DR-white integrations, but, overall, there were no significant differences in HR frequencies observed for euchromatic and heterochromatic insertions. The number of germline SSA events in heterochromatic DR-white insertions was relatively low $16 \%-$ $8 \%$ ) and was comparable with numbers observed at a euchromatic DR-white locus (6\%) (Supplemental Fig. S3A; Do et al. 2014). We conclude that, in the male germline, DSB repair in heterochromatin can occur through HR and, to a lesser extent, SSA.

The contribution of NHEJ in DSB repair cannot be readily determined by assessing the eye color of the offspring (Fig. 1A). In addition, we wished to obtain a comprehensive overview of repair events in somatic cells, not only the germline. Therefore, we induced breaks in flies con- taining DR-white and I-SceI transgenes and subsequently PCR-amplified and Sanger-sequenced the upstream white gene from whole larval genomic DNA (Supplemental Fig. S3B). This allowed for the identification of HR and NHEJ repair events (limited to small insertions and deletions [indels]) but excluded SSA events. The number of identified repair products, determined using the established TIDE (tracking of indels by decomposition) algorithm (Brinkman et al. 2014), varied among integration sites $(16 \%-40 \%)$, possibly reflecting different efficiencies of I-SceI cutting (Supplemental Fig. S3C,D). However, this variability was not directly correlated with DR-white insertions in either euchromatin or heterochromatin and is therefore not simply explained by reduced repair efficiency or reduced accessibility of I-SceI cut sites in heterochromatin. In addition, this result shows that temporal induction of I-SceI expression using either the ecDHFR or hsp70 system generates a sufficient amount of repair products for further detailed analysis.

Next, we determined the proportion of HR and NHEJ products in somatic cells and identified the exact sequences present in all NHEJ products using Illumina sequencing of DR-white PCR products. This revealed that euchromatic and heterochromatic DSBs generate both HR and NHEJ repair products (Fig. 3B,C). Surprisingly, only $14 \%-35 \%$ of identified repair products resulted from HR (Fig. 3B). In contrast, $65 \%-86 \%$ of identified repair products contained small indels, demonstrating that the majority of DSBs are repaired by NHEJ (Chiruvella et al. 2013). Sanger sequencing and TIDE analysis (Supplemental Fig. S3E) revealed similar levels of HR and NHEJ products at specific DR-white integrations when compared with Illumina sequencing (cf. Fig. 3B and Supplemental Fig. S3E).

The majority (99\%-100\%) (Fig. 3C) of NHEJ products analyzed contained small, 1- to 4-base-pair (bp) deletions, with 1-bp deletion products as the most abundant (Supplemental Fig. S3F). Further analysis revealed that $1.5 \%-4 \%$
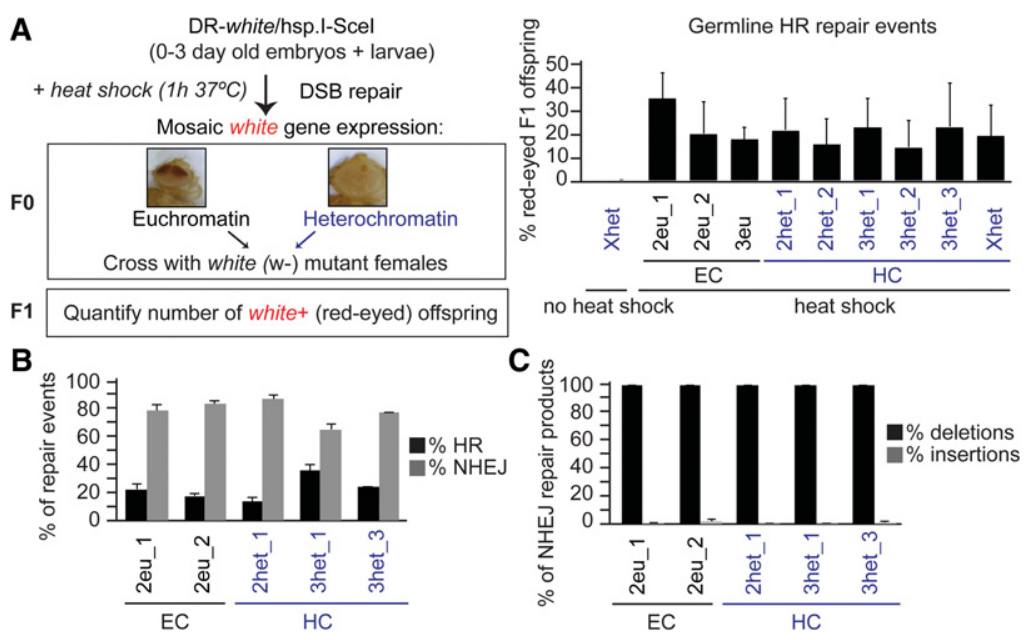

Figure 3. Sequence analysis of repair products reveals utilization of both HR and NHEJ pathways in heterochromatin $(\mathrm{HC})$ and euchromatin $(\mathrm{EC})$. $(A$, left) Schematic of crosses performed for DR-white germline repair product analysis. DR-white/hsp 70 .I-SceI embryos and larvae (0-3 d old) were heatshocked for $1 \mathrm{~h}$ at $37^{\circ} \mathrm{C}$ to induce I-SceI expression and single DSBs and then allowed to develop into adults. Male DR-white/hsp70.I-SceI adults with mosaic white expression were crossed to white mutant (-) females, and F1 progeny were analyzed for eye color. (Right) The percentage of red-eyed (white ${ }^{+}$) F1 progeny (HR events) in the germline is plotted for the indicated DR-white insertions. $n \geq 7$ F0 DRwhite/hsp70.I-SceI males per DR-white insertion. (B) Illumina sequencing of the upstream white PCR product from genomic DNA of the indicated DRwhite/ecDHFR-I-SceI larvae that were fed trimethoprim. The percentage of reads with insertions and deletions (NHE); grey bars) or HR products (HR; black bars) over the total pool of reads of detectable repair products is plotted. Bars indicate average percentages + SD of two independent experiments (larvae) per condition. (C) Quantification of the percentage of NHEJ products with deletions (black) or insertions (gray) identified by Illumina sequencing for the indicated DR-white insertions. Bars indicate average percentages $+\mathrm{SD}$ of two independent experiments (larvae) per condition. 
of these deletion products contained microhomologies of 2 to $>4$ bp (Supplemental Fig. S3G), suggesting that microhomology-mediated Alt-EJ (MMEJ) could play a minor role in both euchromatin and heterochromatin repair.

Cell cycle differences between animals with different DR-white integration sites could potentially affect the repair pathway analysis. However, comparing cell cycle profiles of actively dividing larval wing discs with both heterochromatic and euchromatic DR-white insertions (two each) using the Fly-FUCCI system (Supplemental Fig. S4A; Zielke et al. 2014) did not reveal any overt cell cycle differences that could impact results from the sequence analysis.

In order to confirm the role of HR and NHEJ proteins in DSB repair in euchromatin and heterochromatin, we performed RNAi-mediated knockdown of DmKu70 (Fig. 4A), DmRad51 (Fig. 4B), or DmCtIP (Fig. 4C) in DR-white/ISceI larvae and analyzed the repair products. DmRad51 or DmCtIP depletion reduced the proportion of TIDEidentifiable HR events (Fig. 4B,C) for euchromatic and heterochromatic DR-white integrations, confirming roles for HR in both chromatin compartments. However, knockdown of DmRad51 or DmCtIP also significantly reduced the total amount of repair products in two of three and one of two DR-white insertions, respectively (Supple-

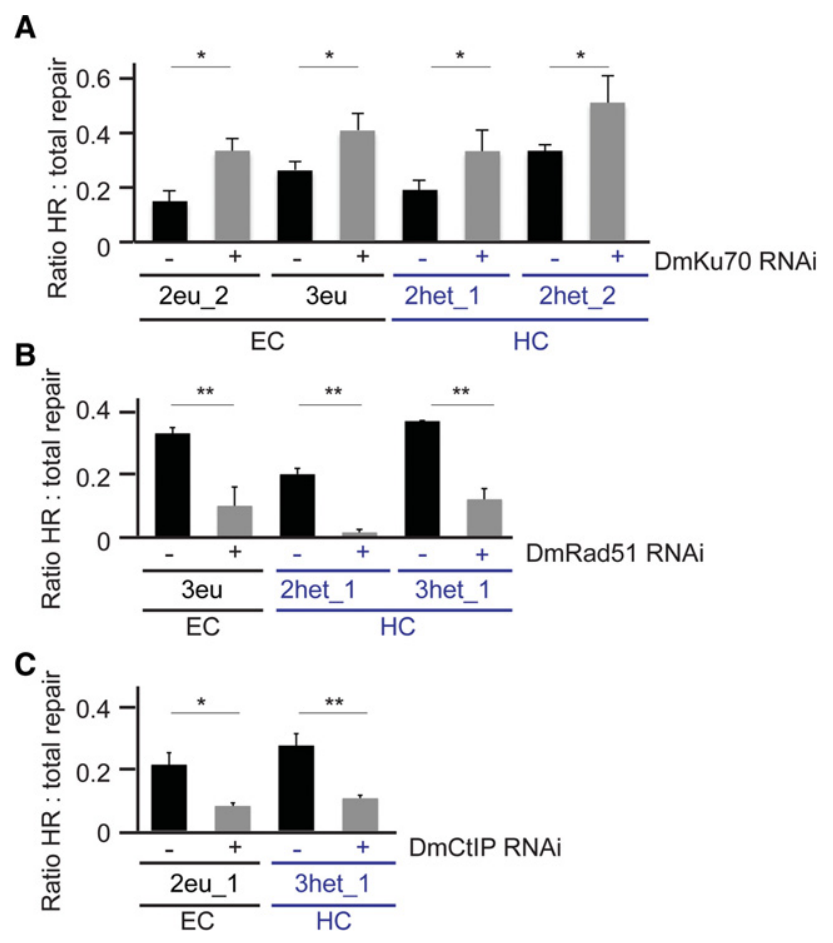

Figure 4. Heterochromatic (HC) and euchromatic (EC) DSB repair uses both NHEJ and HR pathways. $(A-C)$ Quantification of the relative ratio of $\mathrm{HR}$ products over total identified repair products (+SEM) using the TIDE algorithm in the presence (black bars; GAL4 only) or absence (gray bars; GAL4 + UAS.RNAi) of DmKu70 $(A), \operatorname{DmRad} 51(B)$, or DmCtIP $(C) . n \geq 3$ DR-white/ $I$-SceI larvae per condition. (*) $P$-value $\leq 0.04$; (**) $P$-value $\leq$ 0.009 , two-tailed unpaired Student's $t$-test. mental Fig. S4B,C). This suggests that, in the absence of DmRad51 or DmCtIP, DSBs either remain unrepaired or employ an alternative pathway (e.g., SSA) (Do et al. 2014), which is undetectable in this PCR sequence analysis.

We also observed that DmKu70 depletion significantly increased the proportion of HR products (Fig. 4A) and decreased the proportion of indels (NHEJ) (Supplemental Fig. S4D,E). Interestingly, in contrast to depletion of HR proteins, loss of DmKu70 was not accompanied by a significant decrease in the total amount of identified repair products (Supplemental Fig. S4D,E). This result suggests that euchromatic and heterochromatic DSBs can be repaired by $\mathrm{HR}$ in the absence of a functional NHEJ pathway.

Overall, the frequencies of HR and NHEJ repair products as well as dependency on canonical HR and NHEJ proteins were similar for both euchromatic and heterochromatic DR-white insertions. Thus, we conclude that HR and NHEJ are used for DSB repair in both euchromatic and heterochromatic regions. Finally, DSBs in both chromatin domains can use HR when NHEJ is inhibited; however, further studies are needed to determine whether other repair pathways are used upon loss of HR proteins.

Homologous chromosomes are paired in the presence and absence of DSB induction but infrequently serve as a template for $H R$ repair

HR repair in the DR-white reporter system could involve recombination with homologous sequences in cis using the downstream iwhite sequence or in trans using white or iwhite on the homolog or sister chromatid. In Drosophila, homologous chromosomes are paired throughout the cell cycle (Fig. 5A; McKee 2004). The homolog can be used efficiently as a template for HR in the Drosophila germline (Rong and Golic 2003), while its use is more limited in both mammals and yeast (Kadyk and Hartwell 1992; Liang et al. 1998; Johnson and Jasin 2000).

In order to evaluate utilization of the homologous chromosome as a template for HR repair in both euchromatin and heterochromatin, we first determined whether homologs remain paired after DSB induction. This was addressed by generating fly lines containing 256 LacO repeats next to the I-SceI cut site in the maternal homolog (LacO.I-SceI $I_{\text {cut }}$ ) and 256 TetO repeats (without I-SceI cut site) in the paternal homolog (TetO) at the same genomic locus (one heterochromatic and two euchromatic integrations) (Fig. 5B). FISH with TetO and LacO probes showed that, in actively dividing larval discs, the centroids of the TetO and LacO signals for both euchromatic and heterochromatic loci were separated by $\sim 300 \mathrm{~nm}$ in the absence or presence of DSBs ( $\gamma \mathrm{H} 2 \mathrm{Av}$ foci) (Fig. 5C). Thus, homologs remain closely associated after DSB induction and could potentially be used as a template for HR in both euchromatin and heterochromatin.

We assessed the utilization of homologous sequences on the sister chromatid (or in cis intrachromosomal templates) versus sequences on the homolog by generating flies containing only the iwhite gene (iwhite_SNP) plus 
A

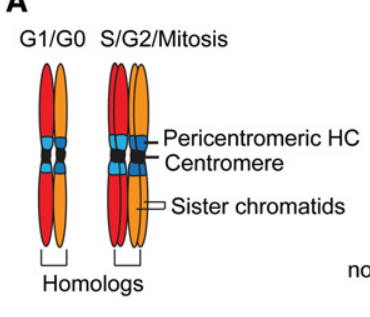

D

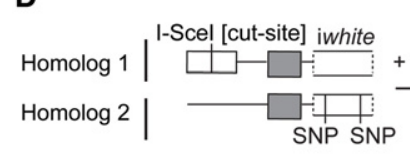

E

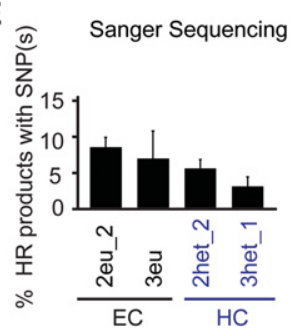

B
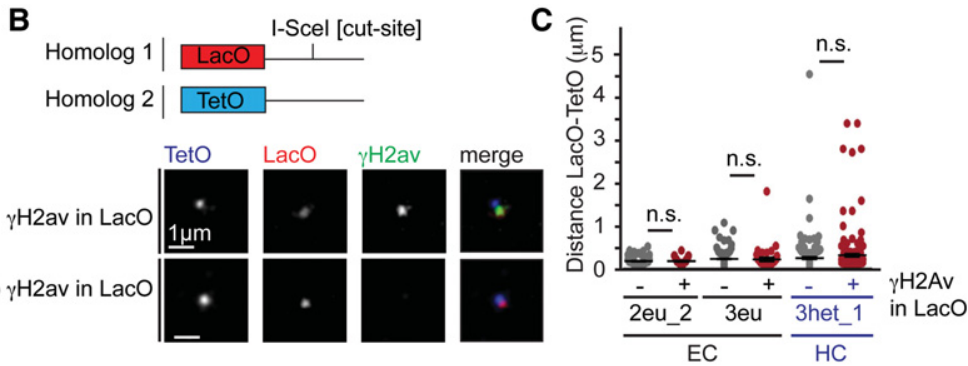

$\mathbf{G}$
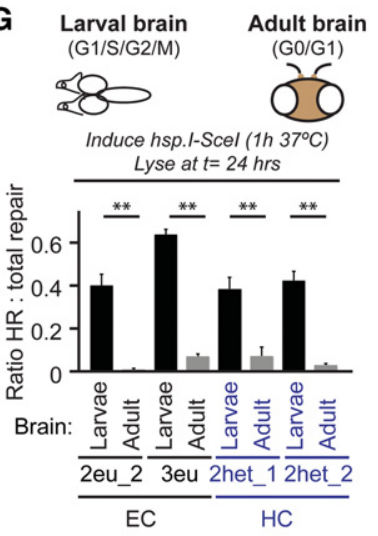

Figure 5. Recombination with the homologous chromosome. (A) Schematic of homolog pairing in Drosophila in the G1/S/G2 phases of the cell cycle. (B, top) Schematic of the LacO.I-SceI[cut-site]/TetO system. 256xLacO.I-SceI[cut-site] (red) insertion on one homolog and 256xTetO (blue) on the other homolog allow for the visualization of the two homologs. (Bottom) Representative images of third instar leg disc cells stained with $\mathrm{LacO}$ (red) and TetO (blue) FISH probes in combination with $\gamma \mathrm{H} 2 \mathrm{Av}$ (green) immunofluorescence in the absence $(-\gamma \mathrm{H} 2 \mathrm{Av})$ and presence $(+\gamma \mathrm{H} 2 \mathrm{Av})$ of a single DSB. (C) Quantification of images as shown in $B$. Distance (in nanometers) between the LacO and TetO FISH centroids is shown in the absence $(-\gamma \mathrm{H} 2 \mathrm{Av}$ in $L a c O$; gray dots) or presence of a single DSB $(+\gamma \mathrm{H} 2 \mathrm{Av}$ in $L a c O$; red dots). One dot represents one cell with a $L a c O$ and TetO signal. $n \geq 50$ cells per condition. The black line indicates mean \pm SEM. (n.s.) Not significant $(P$-value $\geq 0.11)$, unpaired two-tailed Student's $t$-test. $(D) \mathrm{HR}$ with the sister chromatid or in cis (intrachromosomal) in the DR-white) iwhite single-nucleotide polymorphism (SNP) system results in a wild-type white gene (shown at the top). HR with the homolog in the DR-white/iwhite_SNP system results in a white gene containing one or two SNPs, which are 15 bp upstream of and 13 bp downstream from the iwhite site homologous to the I-SceI cut site. (E) Quantification of the percentage of HR products with SNPs (HR with the homolog) of the total pool of HR sequences (+SEM) using TIDE-Sanger sequencing analysis of the indicated DR-white/iwhite_SNP integrations. $n \geq 6$ flies per condition. (F) Quantification of the number of reads containing HR products with SNPs (HR with the homolog; red) and wild-type white (HR with sister or in cis; black) by Illumina sequencing (+SD). $n=2$ larvae per condition. (G, top) Schematic representation of single DSB induction in larval (left) and adult (right) brains. Twenty-four hours after heat-shock induction of hsp70.I-SceI, brains were processed for PCR amplification and Sanger sequence analysis using TIDE. (Bottom) Bars represent the average ratio of HR products over total identified repair products of three independent experiments (+SEM) for larval (black) and adult (gray) brains with the indicated DR-white insertions. $n \geq 3$ brains per condition. $\left(^{* *}\right) P$-value $\leq 0.0012$, unpaired two-tailed Student's $t$-test.

two silent SNPs (single-nucleotide polymorphisms) inserted $15 \mathrm{bp}$ upstream of and $13 \mathrm{bp}$ downstream from the I-SceI cut site, respectively (Fig. 5D). Crossing iwhite_SNP flies with flies containing a DR-white integration at the exact same genomic locus results in progeny with DR-white on one homolog and iwhite_SNP on the other homolog (Fig. 5D). Recovery of DR-white HR products containing the SNPs identifies HR events with the homolog. We used specific amplification of the upstream white gene from DR-white/iwhite_SNP flies expressing ecDHFR.I-SceI and Sanger sequencing to determine the presence of HR sequences with and without the SNPs (Fig. 5D,E). This analysis revealed that the homolog is used in only $3 \%-10 \%$ of all HR repair events, depending on the integration site (Fig. 5E). Illumina sequencing of the upstream white gene in repair products from one eu- chromatic (2eu_2) and one heterochromatic (3het_1) insertion produced the same frequencies observed with Sanger sequencing (Fig. 5F). We conclude that HR with the sister chromatid (or in cis) is strongly preferred for both heterochromatic and euchromatic DSBs despite constitutive homolog pairing.

This led us to determine whether the homolog might be favored as a template for HR repair in the absence of a sister chromatid. In most organisms, there is limited HR in the G1 phase of the cell cycle, until S-phase replication produces sister chromatids (Ciccia and Elledge 2010). However, constitutive homolog pairing (McKee 2004) as well as the presence of many repetitive sequences in Drosophila heterochromatin suggest that HR repair could potentially occur in G0 or G1. To test this hypothesis, we compared the frequencies of DR-white/iwhite_SNP HR 
products in mitotically active larval brains with adult brains, which are mostly composed of differentiated (G0/G1) cells (Fig. 5G). We heat-shock-induced hsp70.ISceI and harvested adult or larval brains $24 \mathrm{~h}$ later. Strikingly, the proportion of repair events that used HR was significantly lower in the adult brains compared with larval brains, while the total amount of identified repair events was similar between the two tissues in three of four DRwhite insertions tested (Supplemental Fig. S5). One DRwhite insertion (2het_1) showed a small but significant reduction in the total amount of repair products in adults $(18 \%)$ compared with larval brains $(23 \%)$, possibly reflecting a reduced induction of hsp70.I-SceI in adult brains, decreased DSB repair, or the use of an undetected alternative repair pathway. Nevertheless, $\mathrm{HR}$ is inhibited in G0/G1 cells that lack sister chromatids despite the presence of paired homologs (Fig. 5G). The levels of HR in adult brains were below the detection limit needed to evaluate the presence of the SNPs (HR with the homolog), precluding comparisons with homolog HR frequencies determined for cycling larval tissues (Fig. 5E,F). We conclude that levels of HR with the homolog remain low in the G0 and G1 cell cycle phases, where we observed that NHEJ is the preferred pathway for DSB repair in both euchromatic and heterochromatic regions.

\section{Discussion}

Here we describe the successful integration and use of a single DSB system in Drosophila heterochromatin and euchromatin, which allowed us to analyze live DSB spatial dynamics and kinetics. To assess utilization of the two main repair pathways (HR and NHEJ), we also performed a detailed sequence analysis of DSB repair products from euchromatic and heterochromatic sites. We conclude that both chromatin regions employ these two repair pathways at similar frequencies, with NHEJ being the most prominent repair pathway used. In addition, we show that the homologous chromosome can be used as a template for HR repair, but sister chromatid (or in cis) sequences are preferred. Finally, HR levels drop significantly in differentiated adult brains, revealing cell cycle regulation in Drosophila that is similar to that in many other organisms independently of whether the DSB is located in heterochromatin or euchromatin.

Mobility of DSBs has been observed at both euchromatic and heterochromatic sites (Chiolo et al. 2013; Dion and Gasser 2013). However, the movement of heterochromatic foci is distinct. Heterochromatic DSBs initiate early repair events, move outside the heterochromatin domain, and ultimately associate with the nuclear periphery, where they recruit Rad51 and continue HR repair (Chiolo et al. 2011; Ryu et al. 2015). We observed that the movement of multiple heterochromatic DSBs induced by IR in cultured cells is also displayed by single DSBs in animal tissues. Thus, the distinct spatio-temporal responses at heterochromatic DSBs occur independently of the number or type of DSBs induced and, importantly, in animal tissues and not only cultured cells. Furthermore, this sug- gests that other damaging events induced by IR, such as oxidative stress, are not the main cause of heterochromatic DSB relocalization.

The observations that (1) the majority of heterochromatic DSBs are repaired by NHEJ, (2) $70 \%$ of breaks become associated with the heterochromatin periphery, and (3) repair focus movement was unaltered after either DmRad51, DmKu70, or DmCtIP depletion suggest that DSB movement is not limited to breaks undergoing HR repair. We therefore propose that DSB relocalization is an intrinsic, global response for both HR and NHEJ repair in heterochromatin. We previously determined that canonical heterochromatin proteins are important for the peripheral movement of IR-induced DSBs (Chiolo et al. 2011), and it will be important to determine whether DNA damage-specific changes to these proteins (e.g., posttranslational modifications or complex composition) are involved in the movement of heterochromatic DSBs and whether the requirements differ for HR and NHEJ. Indeed, mammalian cell studies suggest that ATM-dependent phosphorylation of Kap1 is important for resolution of DSBs associated with heterochromatin, indicating that specific phosphorylation events could occur in different chromatin regions to promote repair (Goodarzi et al. 2008). In addition, this single-break system will facilitate high temporal and spatial resolution imaging to elucidate the precise chromatin movements and proteins involved in DSB relocalization.

Although heterochromatic DSBs have been suggested to be repaired more slowly compared with euchromatic DSBs in mammals (Goodarzi et al. 2008), we observed no difference in mu2 repair focus kinetics (time from appearance to disappearance) between euchromatic and heterochromatic DSBs (Fig. 2). Previous studies focused on IR-induced breaks (Goodarzi et al. 2008; Noon et al. 2010; Chiolo et al. 2011), which are known to create a variety of DSB ends and other types of DNA damage (Obe et al. 1992). It is therefore possible that these different types of DSB ends could require a longer time for repair, specifically in heterochromatic regions. In addition, the kinetics of break repair might also depend on the specific euchromatic and heterochromatic domains where the break is induced. Application of similar single-break systems is needed to directly address the kinetics and regulation of repair responses in these distinct types of chromatin in mammals.

We previously identified a prominent role for $\mathrm{HR}$ in repair of heterochromatic breaks by observing an accumulation of IR-induced repair foci within heterochromatin after DmRad51 or DmRad54 knockdown in Drosophila cells but not after DmKu70 or DmKu80 depletion /Chiolo et al. 2011). However, in the present study, using live mu2 foci analysis of single DSBs, we observed a delay in repair after DmRad51, DmCtIP, or DmKu70 knockdown, demonstrating that both HR and NHEJ pathways play a role in the timely repair of heterochromatic breaks (Fig. 2). More importantly, DNA sequence analysis revealed that NHEJ is used more frequently than $\mathrm{HR} / \sim 80 \%$ vs. $\sim 20 \%$, respectively) for all tested heterochromatic and euchromatic DR-white insertions (Fig. 3). 
A possible explanation for this difference is that our previous study used IR and fixed Drosophila cultured cells. The variety and multiple number of DNA breaks induced by IR (Obe et al. 1992) could require different repair pathways and depend more on HR specifically in heterochromatin. In contrast, the relatively simple single breaks produced by I-SceI are more similar to DSBs induced by replication fork collapse and, with limited processing, might be more easily repaired by NHEJ. In addition, the percentage of cultured Drosophila cells in S and G2 is higher (>95\%) (Chiolo et al. 2011) than in the larval tissues analyzed here ( $50 \%$ ) (Supplemental Figs. S1D, S4A), which could explain the observed differences in repair pathway utilization. Regardless, the more direct and extensive analyses presented here provides definitive evidence that repair of a simple DSB in whole tissues occurs via NHEJ more frequently than HR repair for both euchromatic and heterochromatic DSBs.

Previous studies using DSB reporters in Drosophila have found relatively low levels of NHEJ utilization for DSB repair in euchromatic regions and high levels of SSA (Rong and Golic 2003; Preston et al. 2006; JohnsonSchlitz et al. 2007; Do et al. 2014). In addition, we identified a relatively low $(3 \%-10 \%)$ usage of the homolog as a template for HR (Fig. 5E,F), in contrast to a male germline repair study that identified $\sim 45 \%$ of $\mathrm{HR}$ with the homolog (Rong and Golic 2003). One major difference between our study and other published studies is that the DR-white system introduces multiple in cis and in trans HR templates, which could compete with use of the homolog and reduce the number of identified HR events with the homolog.

Another important issue that can account for the observed differences in repair pathway dependency and homolog utilization is that here we mostly analyzed somatic cell repair products in the whole animal, in contrast to the exclusive focus on premeiotic male germline events in the other studies. Variations in chromatin signatures as well as cell cycle differences in whole larvae versus premeiotic germline cells could account for different repair outcomes. In addition, all germline studies are limited to phenotypic analyses of offspring, which may not provide as much information as repair product sequence analysis.

Although we found that the majority of heterochromatic DSBs use either the HR or NHEJ pathway, we cannot rule out the involvement of other pathways in heterochromatin DSB repair. The percentage of identified SSA events in heterochromatin in germline repair experiments was relatively low $(6 \%-8 \%)$ and comparable with euchromatic DSBs (Supplemental Fig. S3A; Do et al. 2014). Other studies using DSB repair reporters revealed a more prominent role for SSA in euchromatic DSB repair in Drosophila, ranging from $45 \%$ to $85 \%$, depending on the reporter (e.g., length of resection required for annealing) and repair templates present (Rong and Golic 2003; Preston et al. 2006; Johnson-Schlitz et al. 2007). The presence of an HR repair template in the vicinity of the DSB can efficiently compete with SSA for repair (Rong and Golic
2003). Therefore, the multiple HR repair templates present in the DR-white reporter system used here (Fig. 1A; Do et al. 2014) could reduce SSA utilization when compared with other reporter systems.

Nevertheless, in the absence of effective NHEJ or HR repair, alternative pathways such as SSA or Alt-NHEJ/ MMEJ might become more important (Chan et al. 2010; Do et al. 2014; Ceccaldi et al. 2015). We observed that inhibition of NHEJ results in increased utilization of HR without a decrease in the total number of repair events (Fig. 4; Supplemental Fig. S4). However, it is unclear whether pathway "switching" can occur when HR is inhibited, since depletion of DmRad51 and DmCtIP reduced the total identified repair events in some but not all sequenced samples (Supplemental Fig. S4B,C). Thus, alternative repair pathways whose products could not be identified with these methods could repair DSBs in the absence of an active HR pathway. These alternative pathways are known to cause structural chromosomal changes (Chiruvella et al. 2013) and therefore might be more prone to induce aberrant repair products that could severely affect chromosome structure (e.g., dicentrics and acentrics). It will be of interest to analyze the role of alternative repair pathways in distinct chromatin regions and determine whether there are differences with respect to aberrant repair in the absence of canonical HR and NHEJ pathways.

Our results require refinement of our model for how DSB spatio-temporal dynamics and heterochromatin proteins contribute to repairing heterochromatic DSBs in a manner that ensures genome stability. First, the demonstration in this study that homologs are infrequently used for HR repair even in noncycling cells suggests that in cis (e.g., tandem repeats) or sister chromatid exchange events are more likely to be used for "safe" HR repair. Second, the identification of a major role for NHEJ in heterochromatin DSB repair suggests an additional mechanism for ensuring genome stability. Specifically, NHEJ could circumvent the harmful chromosome aberrations that result from recombination between repeats on nonhomologous chromosomes and avoid consequences of recombination with sister chromatid or in cis templates, which result in DNA loss or gain and generation of extra chromosomal circles (Peng and Karpen 2007).

In sum, we developed a targeted single-break system in specific heterochromatic regions and confirmed that single DSBs in heterochromatin display spatio-temporal dynamics in in vivo larval tissue similar to those previously observed for multiple DSBs after IR exposure in cultured cells. In addition, we revealed a prominent role for both the NHEJ and HR repair pathways in DSB repair independently of whether the break is induced in euchromatic or heterochromatic regions. Future research using this single-break system will facilitate more detailed investigations of how DSB repair is regulated in these distinct nuclear compartments (including the roles of chromatin dynamics and developmental stages) and how disease states associated with genome instability are impacted by defects in heterochromatin DSB repair. 


\section{Materials and methods}

Fly lines and genotyping

Flies were grown at room temperature on standard medium, except where otherwise noted. Embryo injections and generation

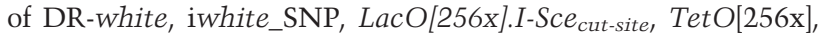
eYFP-mu2, and ecDHFR-HA.I-SceI fly lines were performed by BestGene, Inc. An overview of the MiMIC integration sites and primers used for creating and genotyping DR-white, iwhite_SNP,

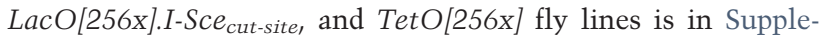
mental Table S1. yw; DR-white.y+ (DR-white_2eu_1), yw; hsp70.HA.I-Sce,Sco/CyO, and yw; +; hsp70.HA.I-Sce,Sb/TM6B, $\mathrm{Tb}, \mathrm{Hu}$ flies were described previously (Do et al. 2014). Transgenic fly lines expressing RFP-HPla from its endogenous promoter were a kind gift from Dr. Hong Wen (Wen et al. 2008). The RNAi lines used were UAS-DmKu70 RNAi (Bloomington Drosophila Stock Center, 29594), UAS-DmRad51 RNAi (Vienna Drosophila Resource Center, 13362), UAS-DmCtIP (CG5872) RNAi (Bloomington Drosophila Stock Center, 36622; Vienna Drosophila Resource Center, 100035), and UAS.vermillion RNAi (Bloomington Drosophila Stock Center, 50641). Act5C-Gal4 transgenes integrated on chromosome 2 (Bloomington Drosophila Stock Center, 4414) or chromosome 3 (Bloomington Drosophila Stock Center, 3954) were used for driving UAS.RNAi expression. Fly-FUCCI integrated on chromosome 3 (Bloomington Drosophila Stock Center, 55124) was used for cell cycle analysis. Genotyping was performed by lysing flies or larvae using the Phire animal tissue direct kit (Thermo Fisher) according to the manufacturer's guidelines. Information on the cloning of constructs and plasmids is in the Supplemental Material.

\section{$D R$-white repair analysis}

Quantification of germline DR-white repair products was performed as previously described (Do et al. 2014). SSA PCR was performed using primer sets described in Supplemental Table S1. Quantification of somatic repair products in DR-white ${ }^{+}$ ${\mathrm{I}-S c e I^{+}}$larvae was performed by inducing I-SceI expression in larvae.

ecDHFR system DR-white females were crossed with ecDHFR-ISceI males on food containing trimethoprim, which allows first through third instar DR-white; ecDHFR-I-SceI larval progeny to consume food containing $40 \mu \mathrm{M}$ trimethoprim and stabilize ecDHFR-I-SceI protein. Third instar DR-white; ecDHFR-I-SceI larvae were harvested and lysed for sequence analysis. To prepare food, $1 \mathrm{~g}$ of Carolina Biological blue food (Formula 4-24 Instant Drosophila medium; blue) was mixed with $3 \mathrm{~mL}$ of nondistilled water containing $3.2 \mu \mathrm{L}$ of $100 \mathrm{mM}$ trimethoprim while vortexing.

Hsp70.I-SceI system DR-white females were crossed with hsp70.I-SceI males. The DR-white/hsp70.I-SceI second instar larvae were heat-shocked for $1 \mathrm{~h}$ at $37^{\circ} \mathrm{C}$ and then harvested and lysed for sequence analysis after $24 \mathrm{~h}$. A detailed explanation of the sequencing analysis is in the Supplemental Material.

\section{FISH, EdU, and immunofluorescence (IF) staining}

For IF, FISH, and FISH-IF stainings, imaginal discs were fixed as described previously (Dernburg 2012) and stored at $-20^{\circ} \mathrm{C}$ in $95 \%$ ethanol. For all stainings, slides were thawed at room temperature and washed (dehydrated) in PBS for $20 \mathrm{~min}$. Detailed pro- tocols for IF, EdU staining, FISH, and FISH-IF as well as the generation of FISH probes are in the Supplemental Material.

\section{Imaging}

Images of wing, leg, or eye disc cells were acquired using a $60 \times$ oil immersion objective (N.A. 1.40) on a Deltavision microscope (Deltavision Spectris; Applied Precision, LLC), and images were deconvolved using SoftWoRx (Applied Precision, LLC). Timelapse images were acquired once every $10 \mathrm{~min}$. Image analysis and focus tracking of deconvolved images was performed manually using Fiji image analysis software.

For live mu2 foci tracking or Fly-FUCCI analysis, third instar discs were pipetted on a slide in $10 \mu \mathrm{L}$ of Schneider S1 medium supplemented with $10 \%$ FBS. A $22 \times 22$-mm number 1.5 coverslip (VWR) was placed on top of the discs, as described before (Lerit et al. 2014). For mu2 focus tracking, the medium was supplemented with $400 \mu \mathrm{M}$ trimethoprim.

\section{ChIP- $q P C R$}

Larvae were ground using a mortar and pestle and liquid nitrogen. The powder was homogenized using a pestle $\mathrm{A}$ and $\mathrm{B}$ glass douncer in PBS 1 mM EDTA (pH 8.0). Fixation, nuclear extraction, and sonication of third instar larval tissues were subsequently performed as described previously (Riddle et al. 2011). ChIP was performed as described elsewhere (O'Geen et al. 2011) using $3 \mu \mathrm{g}$ of H3K9me3 or H3K36me3 antibody and $2 \mu \mathrm{g}$ of third instar larval chromatin. Enrichment for H3K9me3 and H3K36me3 was quantified by qPCR using absolute blue qPCR SYBR low ROX mix (Thermo-Fisher Scientific) and primers specific for the I-SceI (cut site) as well as the yellow (H3K9me3) or rp49 (H3K36me3) gene as an internal control. qPCR was performed on the 7500 Fast real-time PCR system (Applied Biosystems). Primer sequences are in Supplemental Table S1.

\section{$R T-P C R$}

RNA was isolated by homogenizing single larvae in $200 \mu \mathrm{L}$ of Trizol. After addition of $60 \mu \mathrm{L}$ of chloroform and centrifugation, RNA from the aqueous phase was purified using an RNAeasy kit (Qiagen). cDNA was synthesized using SuperScript III (Invitrogen) and oligo dT primers (IDT) following standard cDNA synthesis protocol (Invitrogen). PCR was subsequently performed on the cDNA with gene-specific primers (Supplemental Table S1).

\section{Immunoblotting}

Larvae were lysed in $30 \mu \mathrm{L}$ of lysis buffer $(20 \mathrm{mM}$ TrisHCl, $10 \mathrm{mM}$ $\mathrm{KCl}, 1.5 \mathrm{mM} \mathrm{MgCl}_{2}, 200 \mathrm{mM} \mathrm{NaCl}, 0.1 \% \mathrm{NP} 40$ ) for 30 min at $4^{\circ}$ $\mathrm{C}$ and subsequently boiled for $5 \mathrm{~min}$ at $95^{\circ} \mathrm{C}$ in the presence of SDS loading buffer ( $5 \times$ diluted). Ten microliters of the total lysate was separated by SDS-PAGE and transferred to nitrocellulose membranes (Whatman), which were blotted with primary antibodies overnight at $4^{\circ} \mathrm{C}$. Peroxidase-coupled or fluorescently labeled secondary antibodies and ECL (GE Healthcare) or Odyssey scanning (Li-cor) were used to visualize protein bands, respectively.

\section{Antibodies}

Primary antibodies used for Western blotting were rabbit antiDmKu70 (1:1000; a gift from Dr. Donald Rio), rabbit antiDmRad51 (1:2000; a gift from Jim Kadonaga), and mouse anti- 
tubulin (1:5000; Sigma). Primary antibodies used for IF were mouse anti-HA (1:1000; ab130275), rabbit anti- $\gamma \mathrm{H} 2 \mathrm{av}$ (rabbit [1:250; Rockland] or mouse [1:250; Developmental Studies Hybridoma Bank, UNC93-5.2.1]), mouse anti-Cyclin A (1:10; Developmental Studies Hybridoma Bank, A12), rabbit anti-Ser10 phospho Histone H3 (1:1000; Upstate Biotechnology, 06-570), and rabbit anti-cleaved caspase3-Asp175 (1:500; Cell Signaling Technology, 9661). ChIP antibodies used were rabbit antiH3K36me3 (ab9050) and rabbit anti-H3K9me3 (ab8898). Secondary antibodies used were Alexa 568 goat anti-rabbit or Alexa 488 goat anti-mouse for IF (1:500; Thermo-Fisher Scientific), goat anti-rabbit-HRP or goat anti-mouse-HRP $(1: 10,000$; ThermoScientific) for ECL, and donkey anti-mouse-680 or donkey antirabbit-800 (1:10,000; Li-cor) for use on the Odyssey (Li-cor).

\section{Acknowledgments}

Special thanks to all members of the Karpen, Dr. Priscilla Cooper, and Dr. Sue Celniker laboratories for their invaluable input during laboratory meetings and project design. These studies were supported by National Institutes of Health grants R01 GM086613 (to G.H.K.) and 1R15GM110454-01 (to J.R.L.), the Dutch Cancer Society (KWF) post-doctoral fellowship 20135854 (to A.J.), and the Netherlands Organization for Scientific Research Rubicon fellowship 825.13.006 (to A.J.). The funders had no role in study design, data collection and analysis, decision to publish, or preparation of the manuscript.

\section{References}

Aymard F, Bugler B, Schmidt CK, Guillou E, Caron P, Briois S, Iacovoni JS, Daburon V, Miller KM, Jackson SP, et al. 2014. Transcriptionally active chromatin recruits homologous recombination at DNA double-strand breaks. Nat Struct Mol Biol 21: 366-374.

Beucher A, Birraux J, Tchouandong L, Barton O, Shibata A, Conrad S, Goodarzi A, Krempler A, Jeggo P, Löbrich M. 2009. ATM and Artemis promote homologous recombination of radiation-induced DNA double-strand breaks in G2. EMBO I 28: 3413-3427.

Brinkman EK, Chen T, Amendola M, van Steensel B. 2014. Easy quantitative assessment of genome editing by sequence trace decomposition. Nucleic Acids Res 42: e168.

Burman B, Zhang ZZ, Pegoraro G, Lieb JD, Misteli T. 2015. Histone modifications predispose genome regions to breakage and translocation. Genes Dev 29: 1393-1402.

Ceccaldi R, Liu JC, Amunugama R, Hajdu I, Primack B, Petalcorin MI, O'Connor KW, Konstantinopoulos PA, Elledge SJ, Boulton SJ, et al. 2015. Homologous-recombination-deficient tumours

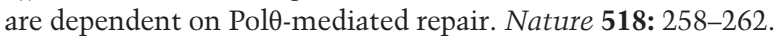

Chan SH, Yu AM, McVey M. 2010. Dual roles for DNA polymerase $\theta$ in alternative end-joining repair of double-strand breaks in Drosophila. PLoS Genet 6: e1001005.

Chen H, Zheng X, Zheng Y. 2014. Age-associated loss of lamin-B leads to systemic inflammation and gut hyperplasia. Cell 159: 829-843.

Chiolo I, Minoda A, Colmenares SU, Polyzos A, Costes SV, Karpen GH. 2011. Double-strand breaks in heterochromatin move outside of a dynamic HPla domain to complete recombinational repair. Cell 144: 732-744.

Chiolo I, Tang J, Georgescu W, Costes SV. 2013. Nuclear dynamics of radiation-induced foci in euchromatin and heterochromatin. Mutat Res 750: 56-66.
Chiruvella KK, Liang Z, Wilson TE. 2013. Repair of double-strand breaks by end joining. Cold Spring Harb Perspect Biol 5: a012757.

Cho U, Zimmerman SM, Chen LC, Owen E, Kim JV, Kim SK, Wandless TJ. 2013. Rapid and tunable control of protein stability in Caenorhabditis elegans using a small molecule. PLoS One 8: e72393.

Ciccia A, Elledge SJ. 2010. The DNA damage response: making it safe to play with knives. Mol Cell 40: 179-204.

Dernburg AF. 2012. Formaldehyde fixation of Drosophila tissues onto slides for whole-mount FISH. Cold Spring Harb Protoc doi: $10.1101 /$ pdb.prot067314.

Dion V, Gasser SM. 2013. Chromatin movement in the maintenance of genome stability. Cell 152: 1355-1364.

Do AT, Brooks JT, Le Neveu MK, LaRocque JR. 2014. Doublestrand break repair assays determine pathway choice and structure of gene conversion events in Drosophila melanogaster. G3 (Bethesda) 4: 425-432.

Dronamraju R, Mason JM. 2009. Recognition of double strand breaks by a mutator protein (MU2) in Drosophila melanogaster. PLoS Genet 5: e1000473.

Eissenberg JC, Elgin SC. 2000. The HP1 protein family: getting a grip on chromatin. Curr Opin Genet Dev 10: 204-210.

Ellinger J, Bachmann A, Goke F, Behbahani TE, Baumann C, Heukamp LC, Rogenhofer S, Muller SC. 2014. Alterations of global histone H3K9 and H3K27 methylation levels in bladder cancer. Urol Int 93: 113-118.

Fodor BD, Shukeir N, Reuter G, Jenuwein T. 2010. Mammalian $\mathrm{Su}(\mathrm{var})$ genes in chromatin control. Annu Rev Cell Dev Biol 26: $471-501$.

Goodarzi AA, Jeggo PA. 2012. The heterochromatic barrier to DNA double strand break repair: how to get the entry visa. Int J Mol Sci 13: 11844-11860.

Goodarzi AA, Noon AT, Deckbar D, Ziv Y, Shiloh Y, Löbrich M, Jeggo PA. 2008. ATM signaling facilitates repair of DNA double-strand breaks associated with heterochromatin. Mol Cell 31: 167-177.

Heitz E. 1928. Das heterochromatin der moose. Jahrb Wiss Bot: 762-818.

Hoskins RA, Carlson JW, Wan KH, Park S, Mendez I, Galle SE, Booth BW, Pfeiffer BD, George RA, Svirskas R, et al. 2015. The Release 6 reference sequence of the Drosophila melanogaster genome. Genome Res 25: 445-458.

Jakob B, Splinter J, Conrad S, Voss KO, Zink D, Durante M, Lobrich M, Taucher-Scholz G. 2011. DNA double-strand breaks in heterochromatin elicit fast repair protein recruitment, histone H2AX phosphorylation and relocation to euchromatin. Nucleic Acids Res 39: 6489-6499.

Janssen A, Medema RH. 2013. Genetic instability: tipping the balance. Oncogene 32: 4459-4470.

Jasin M. 1996. Genetic manipulation of genomes with rare-cutting endonucleases. Trends Genet 12: 224-228.

Johnson RD, Jasin M. 2000. Sister chromatid gene conversion is a prominent double-strand break repair pathway in mammalian cells. EMBO J 19: 3398-3407.

Johnson-Schlitz DM, Flores C, Engels WR. 2007. Multiple-pathway analysis of double-strand break repair mutations in Drosophila. PLoS Genet 3: e50.

Kadyk LC, Hartwell LH. 1992. Sister chromatids are preferred over homologs as substrates for recombinational repair in Saccharomyces cerevisiae. Genetics 132: 387-402.

Kharchenko PV, Alekseyenko AA, Schwartz YB, Minoda A, Riddle NC, Ernst J, Sabo PJ, Larschan E, Gorchakov AA, Gu T, et al. 2011. Comprehensive analysis of the chromatin landscape in Drosophila melanogaster. Nature 471: 480-485. 
Lemaitre C, Grabarz A, Tsouroula K, Andronov L, Furst A, Pankotai T, Heyer V, Rogier M, Attwood KM, Kessler P, et al. 2014. Nuclear position dictates DNA repair pathway choice. Genes Dev 28: 2450-2463.

Lerit DA, Plevock KM, Rusan NM. 2014. Live imaging of Drosophila larval neuroblasts. J Vis Exp doi: 10.3791/51756.

Liang F, Han M, Romanienko PJ, Jasin M. 1998. Homology-directed repair is a major double-strand break repair pathway in mammalian cells. Proc Natl Acad Sci 95: 5172-5177.

Mah LJ, El-Osta A, Karagiannis TC. 2010. $\gamma \mathrm{H} 2 \mathrm{AX}$ : a sensitive molecular marker of DNA damage and repair. Leukemia 24: 679-686.

McKee BD. 2004. Homologous pairing and chromosome dynamics in meiosis and mitosis. Biochim Biophys Acta 1677: $165-180$.

Nagel ZD, Margulies CM, Chaim IA, McRee SK, Mazzucato P, Ahmad A, Abo RP, Butty VL, Forget AL, Samson LD. 2014. Multiplexed DNA repair assays for multiple lesions and multiple doses via transcription inhibition and transcriptional mutagenesis. Proc Natl Acad Sci 111: E1823-E1832.

Noon AT, Shibata A, Rief N, Löbrich M, Stewart GS, Jeggo PA, Goodarzi AA. 2010. 53BP1-dependent robust localized KAP1 phosphorylation is essential for heterochromatic DNA double-strand break repair. Nat Cell Biol 12: 177-184.

Obe G, Johannes C, Schulte-Frohlinde D. 1992. DNA doublestrand breaks induced by sparsely ionizing radiation and endonucleases as critical lesions for cell death, chromosomal aberrations, mutations and oncogenic transformation. Mutagenesis 7: 3-12.

O'Geen H, Echipare L, Farnham PJ. 2011. Using ChIP-seq technology to generate high-resolution profiles of histone modifications. Methods Mol Biol 791: 265-286.

Peng JC, Karpen GH. 2007. H3K9 methylation and RNA interference regulate nucleolar organization and repeated DNA stability. Nat Cell Biol 9: 25-35.

Peng JC, Karpen GH. 2009. Heterochromatic genome stability requires regulators of histone $\mathrm{H} 3 \mathrm{~K} 9$ methylation. PLoS Genet 5: e1000435.

Peters AH, Carroll D, Scherthan H, Mechtler K, Sauer S, Schöfer C, Weipoltshammer K, Pagani M, Lachner M, Kohlmaier A, et al. 2001. Loss of the Suv39h histone methyltransferases impairs mammalian heterochromatin and genome stability. Cell 107: 323-337.

Preston CR, Flores CC, Engels WR. 2006. Differential usage of alternative pathways of double-strand break repair in Drosophila. Genetics 172: 1055-1068.

Price BD, D'Andrea AD. 2013. Chromatin remodeling at DNA double-strand breaks. Cell 152: 1344-1354.

Rangan P, Malone CD, Navarro C, Newbold SP, Hayes PS, Sachidanandam R, Hannon GJ, Lehmann R. 2011. piRNA produc- tion requires heterochromatin formation in Drosophila. Curr Biol 21: 1373-1379.

Riddle NC, Minoda A, Kharchenko PV, Alekseyenko AA, Schwartz YB, Tolstorukov MY, Gorchakov AA, Jaffe JD, Kennedy C, Linder-Basso D, et al. 2011. Plasticity in patterns of histone modifications and chromosomal proteins in Drosophila heterochromatin. Genome Res 21: 147-163.

Rogakou EP, Boon C, Redon C, Bonner WM. 1999. Megabase chromatin domains involved in DNA double-strand breaks in vivo. J Cell Biol 146: 905-916.

Rong YS, Golic KG. 2003. The homologous chromosome is an effective template for the repair of mitotic DNA double-strand breaks in Drosophila. Genetics 165: 1831-1842.

Ryu T, Spatola B, Delabaere L, Bowlin K, Hopp H, Kunitake R, Karpen GH, Chiolo I. 2015. Heterochromatic breaks move to the nuclear periphery to continue recombinational repair. Nat Cell Biol 17: 1401-1411.

Schuster-Bockler B, Lehner B. 2012. Chromatin organization is a major influence on regional mutation rates in human cancer cells. Nature 488: 504-507.

Soong CP, Breuer GA, Hannon RA, Kim SD, Salem AF, Wang G, Yu R, Carriero NJ, Bjornson R, Sundaram RK, et al. 2015. Development of a novel method to create double-strand break repair fingerprints using next-generation sequencing. DNA Repair (Amst) 26: 44-53.

Stucki M, Clapperton JA, Mohammad D, Yaffe MB, Smerdon SJ, Jackson SP. 2005. MDC1 directly binds phosphorylated histone $\mathrm{H} 2 \mathrm{AX}$ to regulate cellular responses to DNA doublestrand breaks. Cell 123: 1213-1226.

Sulli G, Di Micco R, d'Adda di Fagagna F. 2012. Crosstalk between chromatin state and DNA damage response in cellular senescence and cancer. Nat Rev Cancer 12: 709-720.

Venken KJ, Schulze KL, Haelterman NA, Pan H, He Y, EvansHolm M, Carlson JW, Levis RW, Spradling AC, Hoskins RA, et al. 2011. MiMIC: a highly versatile transposon insertion resource for engineering Drosophila melanogaster genes. Nat Methods 8: 737-743.

Weiler KS, Wakimoto BT. 1995. Heterochromatin and gene expression in Drosophila. Annu Rev Genet 29: 577-605.

Wen H, Andrejka L, Ashton J, Karess R, Lipsick JS. 2008. Epigenetic regulation of gene expression by Drosophila Myb and E2F2-RBF via the Myb-MuvB/dREAM complex. Genes Dev 22: 601-614.

You Z, Bailis JM. 2010. DNA damage and decisions: CtIP coordinates DNA repair and cell cycle checkpoints. Trends Cell Biol 20: 402-409.

Zielke N, Korzelius J, van Straaten M, Bender K, Schuhknecht GF, Dutta D, Xiang J, Edgar BA. 2014. Fly-FUCCI: a versatile tool for studying cell proliferation in complex tissues. Cell Rep 7: 588-598. 


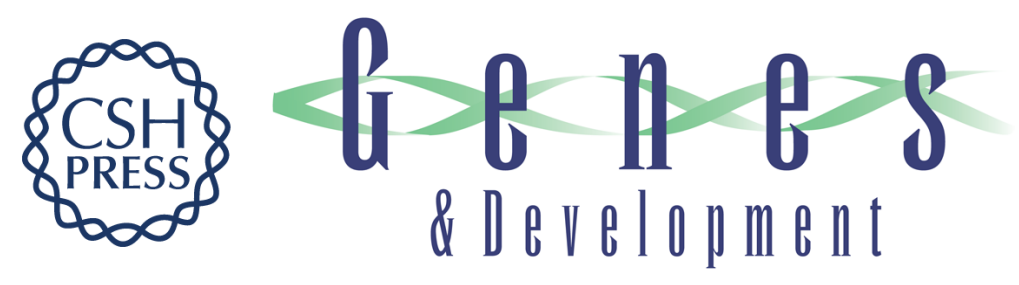

\title{
A single double-strand break system reveals repair dynamics and mechanisms in heterochromatin and euchromatin
}

\author{
Aniek Janssen, Gregory A. Breuer, Eva K. Brinkman, et al.
}

Genes Dev. 2016, 30:

Access the most recent version at doi:10.1101/gad.283028.116

\section{Supplemental http://genesdev.cshlp.org/content/suppl/2016/07/29/30.14.1645.DC1 Material}

References This article cites 57 articles, 16 of which can be accessed free at: http://genesdev.cshlp.org/content/30/14/1645.full.html\#ref-list-1

Creative This article is distributed exclusively by Cold Spring Harbor Laboratory Press for the first Commons six months after the full-issue publication date (see

License http://genesdev.cshlp.org/site/misc/terms.xhtml). After six months, it is available under a Creative Commons License (Attribution-NonCommercial 4.0 International), as described at http://creativecommons.org/licenses/by-nc/4.0/.

Email Alerting Receive free email alerts when new articles cite this article - sign up in the box at the top Service right corner of the article or click here.

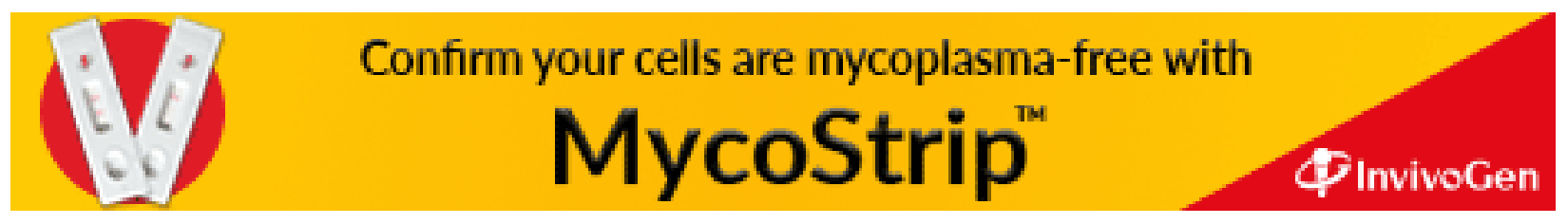

